\title{
Safety for beginners
}

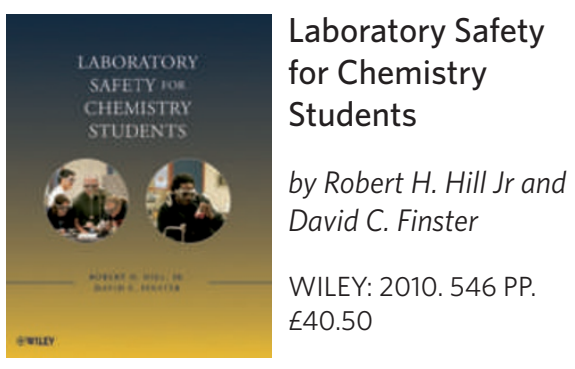

1 afety first' seems to be the motto of every organization that conducts research in chemistry, but seldom in academia does one encounter a department that backs the statement with action. Rather, students are warned of hazards specific to each laboratory exercise, and researchers are given an introductory presentation on safety and left to absorb the rest by osmosis.

In their book Laboratory Safety for Chemistry Students, authors Robert Hill and David Finster offer a more logical and thorough approach. They cover the basics of lab safety by dividing chapters on common subjects into subsections of increasing levels of academic and experimental sophistication. The authors suggest that these sections be assigned to students on an ad hoc basis prior to applicable laboratory experiments over the course of a four-year undergraduate curriculum in chemistry. The requirement of a lesson in safety prior to the start of every lab experiment quite literally puts safety first.

The book covers all of the general topics that one would expect, including personal protective equipment, toxic substances, common hazards, storage and compatibility of chemicals, and emergency response.

The treatment of each subject is straightforward, and in general, isn't especially detailed or specific. For instance, the concept of pyrophoricity is discussed, but no specific instructions are given on how to dispense pyrophoric materials (for example, how to use a syringe or cannula for this purpose).

The authors' treatment of several topics is especially good. For fire safety, they present a nice summary of how fires $\frac{\sim}{\sigma}$ function, a thorough explanation of the classes of fires, and guidance on what types of extinguishers to use in various situations. The discussion of compressed gases is also concise and complete, with a good introduction to using regulators and a helpful rundown of common hazards to avoid. Other subjects could have easily justified more detailed coverage. In the section on personal protective equipment, for example, the importance of wearing eye protection is stated emphatically, with secondary importance placed in donning appropriate gloves and clothing that minimizes the exposure of skin. Although the authors make the point that there is no universal glove for every situation, the reader is referred to a list of online resources to decide what gloves are best for specific classes of chemicals. For such a fundamental topic, I would have preferred a more thorough discussion within the book itself.

Indeed, the book is not without its faults. It's ironic that while reading this treatise on safety, I almost died - of boredom. The book is over 500 pages long, and it's not exactly a page-turner. I would not recommend it to experienced researchers looking for a refresher course in safety; there is simply too much obvious information through which to wade. But students with little or no experience working in labs stand to benefit from exposure to this straightforward material. We must not fall into the trap that what is obvious to us will be obvious to newcomers, especially for important matters of safety.

With that said, I am sceptical as to whether undergraduate students can be counted on to read this book unless coerced by the threat of being quizzed or examined. As stated previously, the text can be dry. Several recurring features of the book serve little value and may annoy readers who would prefer that the authors just get to the point. The authors' favoured approach to safety is embodied in their acronym RAMP (short for recognize, assess, minimize and prepare). The concept is crow-barred into every section of every chapter. Although such an approach seems workable, the idea that readers should consider RAMP first and foremost is not especially compelling.

The authors did manage to spice up their material with stories of laboratory accidents told at the beginning of each chapter. These accounts, with outcomes that range from the benign to the morbid, are a compelling tool for communicating the importance of lab safety. I wish the authors had pursued these vehicles further by actually answering the question posed at the end of each story, "What lessons can be learned from this incident?" Similarly, it would have been nice if answers were provided to the multiple-choice quizzes at th end of each chapter.

\section{The book's greatest contribution is in raising a new idea of how to train undergraduates to work safely in the laboratory.}

Laboratory Safety for Chemistry Students provides a thorough introduction to basic lab safety. Prospective buyers should be aware that this book is nowhere near a comprehensive treatment of lab safety and does not make a suitable desk reference for how to handle specific chemicals or make informed selections of specific safety equipment. Having read the book from cover-to-cover, I think its greatest contribution is in raising a new idea of how to train undergraduates to work safely in the laboratory. I am unaware of the existence of another book that has been designed specifically to be broken down and taught in pieces over the course of a four-year undergraduate curriculum. Although it is easy to hypothesize potential hurdles to the success of this approach, the idea has merit and is worth testing. For instructors willing to run such an experiment, this book provides a solid starting point from which to work.

\section{REVIEWED BY PAUL J. BRACHER}

Paul Bracher is currently a National Science Foundation ACC Postdoctoral Fellow at the California Institute of Technology. 